\title{
Classical mesoscopic effect in the resistance of point contacts
}

\author{
Yu. A. Kolesnichenko ${ }^{1}$, A. N. Omelyanchouk ${ }^{1}$, N. van der Post ${ }^{2}$, \\ and A. I. Yanson ${ }^{1,2}$ \\ ${ }^{1}$ B. I. Verkin Institute for Low Temperature Physics and Engineering of National Academy of Science of Ukraine, \\ 47 Lenin Ave., 310164 Kharkov, Ukraine \\ E-mail: kolesnichenko@ilt.kharkov.ua \\ ${ }^{2}$ Kamerlingh Onnes Laboratorium, Leiden University, P.O. Box 9506, 2300 RA Leiden, The Netherlands \\ E-mail: post@rulgm1.leidenuniv.nl
}

Submitted May 22, 1997

\begin{abstract}
The mesoscopic effect of the dependence of the point-contact conductance on the spatial distribution of the impurities is theoretically studied. It is shown that the resistance dependence on the diameter $d$ is not only determined by the electron mean free path $l_{i}$, but also by the average distance between the impurities. In the case of two types of impurities with different concentrations the mesoscopic effect is predicted for a dirty point contact $\left(d>>l_{i}\right)$ due to the scattering at specific (e.g., magnetic) impurities with a low concentration. Such contacts were numerically modeled for random distributions of the two types of impurities.
\end{abstract}

PACS: 72.15.Qm, 72.10.Fk, 73.40.Jn

The break-junction technique [1] makes it possible to study electron transport through microconstrictions few nanometers in diameter. The conducting properties of such small contacts are affected by quantum effects such as the $2 e^{2} / h$ conductance quantization observed while changing the diameter of the contact [2,3], conductance fluctuations due to electron wave interference $[4,5]$, etc. In ultrasmall contacts, besides the quantum effects [6,7], the presence of impurities in the contact region produces noticeable classic effects. Such «classical mesoscopic effects» cause conductance fluctuations due to the displacement of individual scatterers, and also its stochastic-like dependence on the magnetic field [8]. It was shown in Ref. 9 that individual point defects, which are located at a distance from the constriction comparable to its diameter $d$, cause a deviation of the size-dependence of a ballistic contact's resistance $R(d)$ from Sharvin's formula $[10,11]$. If the average distance between the impurities appears greater than or equal to $d$, we obtain a classical mesoscopic effect - the dependence of additional contact resistance due to the defects on their spatial distribution. We will demonstrate below that an analogous dependence can be also observed in contacts with a short electron mean free path for two types of scatterers of different concentrations. Such a situation can take place, for instance, in experimental observations of size effects in metallic point contacts with magnetic impurities [12], where a rare spin-flip electron scattering is accompanied by many scatterings at regular impurities which inevitably appear at the point contacts.

We restrict the analysis of classical mesoscopic effects to the semiclassical approximation, assuming that the size is considerably larger than the de Broglie wavelength of electrons.

Assuming that the point contact is an orifice of diameter $d$ in the insulating partition $\Sigma$, and separating the two metallic half-spaces, we obtain a situation which is illustrated in Fig. 1. It is assumed that the metal has two types of defects of different effective scattering cross section. The characteristic distance $r_{0}$ between the defects of first type (their concentration is $n_{i}=r_{0}^{-3}$ ) is assumed to be smaller than the contact diameter, which allows us to average all equations over the coordinates of these impurities. We call such defects «the background defects». The concentration of second-type defects $n_{i}^{*}$, whose interaction with electrons determines the 


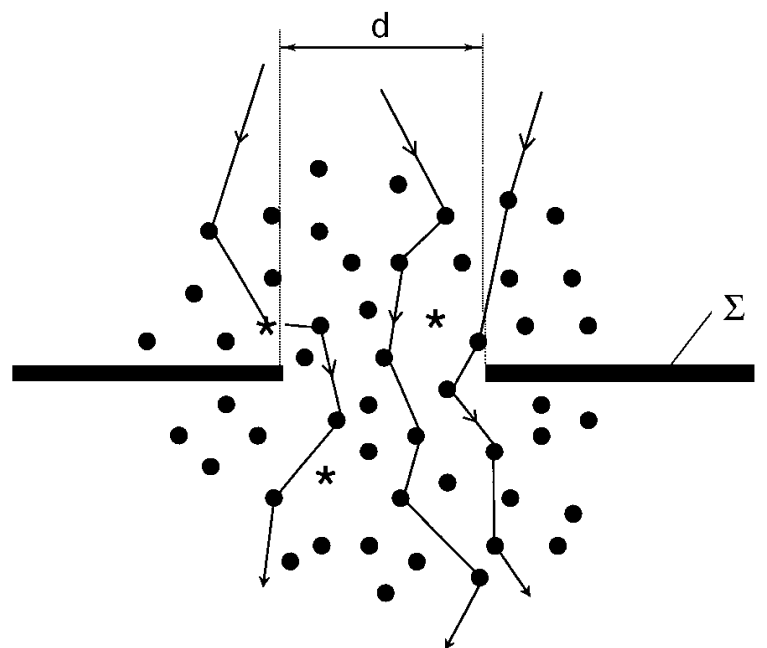

Fig. 1. A model of the point contact as a circular orifice in an insulating partition $\Sigma$. The dots represent the background and the stars symbolize the specific defects. The broken lines represent the electron trajectory through the contact.

mesoscopic effects, is such that their separation, $r_{0}^{*}=n_{i}^{*-1 / 3}$, is comparable to $d$. Such defects are referred to as «specific impurities».

The current through the contact can be expressed in terms of the Green-Keldysh function $g_{\mathbf{p}}^{K}(\mathbf{r}, \varepsilon)$ integrated with respect to the energy variable $\xi=\varepsilon_{\mathbf{p}}-\varepsilon_{F}\left(\varepsilon_{F}\right.$ is the Fermi energy) [13]:

$$
I=\frac{\pi}{2} e N(0) \int_{S} d^{2} \boldsymbol{\rho} \int \frac{d \varepsilon}{2 \pi}\left\langle v_{z} g_{\mathbf{p}}^{K}(\boldsymbol{\rho}, \varepsilon)\right\rangle .
$$

In this formula the integration with respect to $d^{2} \rho$ is carried out over the area $S$ of the contact, and $v_{z}$ is the component of the electron velocity $\mathbf{v}=\partial \varepsilon / \partial \mathbf{p}$ parallel to the contact axis. The angle brackets denote averaging over the directions of the momentum $\mathbf{p}$ at the Fermi surface $\varepsilon_{\mathbf{p}}=\varepsilon_{F}$, where the density of states is $N(0)$.

In the Keldysh method [13] the retarded $g_{\mathrm{p}}^{R}$ and advanced $g_{\mathbf{p}}^{A}$ Green's functions appear along with $g_{\mathbf{p}}^{K}$. The matrix

$$
\hat{g}_{\mathbf{p}}=\left[\begin{array}{cc}
g_{\mathbf{p}}^{A} & g_{\mathbf{p}}^{K} \\
0 & g_{\mathbf{p}}^{R}
\end{array}\right],
$$

which satisfies the normalization condition $\hat{g}_{\mathbf{p}}^{2}=1$ for a nonsuperconducting metal, is the solution of the equation [14]

$$
i \mathbf{v} \nabla \hat{g}_{\mathbf{p}}+\left[\hat{g}_{\mathbf{p}} \Sigma\right]=\left[\hat{t}, \nabla \hat{g}_{\mathbf{p}}^{(0)}\right] .
$$

In this equation $\hat{g}_{\mathbf{p}}^{(0)}$ is the matrix of the Green's function for a pure point contact with only the background impurities, the scattering of electrons on which is described by the coordinate-independent matrix of self-energy functions $\hat{\Sigma}=\left(i / 2 \tau_{i}\right)\left\langle g_{\mathrm{p}}\right\rangle$, where $\tau_{i}$ is the characteristic time of the elastic relaxation. The electron interaction with the specific defects is associated with the right side of Eq. (3) which contains the scattering matrix $\hat{t}$. This matrix can be determined from the Lippman-Schwinger equation

$$
\hat{t}=\hat{V}-i \pi N(0) \hat{V}\left\langle\hat{g}_{\mathbf{p}}^{(0)}\right\rangle \hat{t},
$$

where $\hat{V}=\hat{V}(\mathbf{r})$ is the scattering potential. Note that the structures of matrices $\hat{\Sigma}$ and $\hat{t}$ are similar to that of (2).

According to Eq. (3) and the normalization condition, the functions $g_{\mathbf{p}}^{R, A}$ in a normal metal are equal to their equilibrium values $g_{\mathbf{p}}^{R}=-g_{\mathbf{p}}^{A}=1$. Taking into account relation (4), the equation for $g_{\mathrm{p}}^{K}$ can be written in the form

$$
\mathbf{v} \nabla \hat{g}_{\mathbf{p}}^{K}-I_{i}\left(\hat{g}_{\mathbf{p}}^{K}\right)=I_{i}^{*}\left(\hat{g}_{\mathbf{p}}^{(0) K}\right)
$$

where

$I_{i}\left(\hat{g}_{\mathbf{p}}\right)=\frac{1}{\tau_{i}}\left[\left\langle g_{\mathbf{p}}\right\rangle-g_{\mathbf{p}}\right] ; \quad I_{i}^{*}\left(\hat{g}_{\mathbf{p}}\right)=2 \operatorname{Im} t^{A}\left[\left\langle g_{\mathbf{p}}\right\rangle-g_{\mathbf{p}}\right]$.

The boundary condition

$$
g_{\mathbf{p}}^{K}(\mathbf{r}, \varepsilon)=G_{0}\left(\varepsilon-\frac{1}{2} e U \operatorname{sign} z\right) \text { for }|z| \rightarrow \infty
$$

ensures the restoration of equilibrium in the electron system at the contact banks. In Eq. (6) $G_{0}(\varepsilon)=2 \tanh (\varepsilon / 2 T)$ is the equilibrium Green's function ( $T$ is the temperature), $U$ is the voltage applied to the point contact.

Following the authors of Ref. 15, the function $g_{\mathbf{p}}^{(0) K}(\mathbf{r}, \varepsilon)$ can be conveniently expressed as

$$
\begin{aligned}
& g_{\mathbf{p}}^{(0) K}(\mathbf{r}, \varepsilon)=\alpha_{\mathbf{p}}(\mathbf{r}) G_{0}\left(\varepsilon+\frac{e U}{2}\right)+ \\
& +\left(1-\alpha_{\mathbf{p}}(\mathbf{r})\right) G_{0}\left(\varepsilon-\frac{e U}{2}\right)
\end{aligned}
$$

where the voltage-independent function $\alpha_{\mathbf{p}}(\mathbf{r})$ is the probability that an electron arrives at the point $\mathbf{r}$ from $-\infty$ with the momentum $\mathbf{p}$ after being scattered only by the background impurities. The function $\alpha_{\mathbf{p}}(\mathbf{r})$ satisfies a homogeneous equation similar to the one for $g_{\mathbf{p}}^{(0) K}$

$$
\mathbf{v} \nabla \alpha_{\mathbf{p}}(\mathbf{r})-I_{i}\left(\alpha_{\mathbf{p}}(\mathbf{r})\right)=0
$$

and also the condition for $|\mathbf{r}| \rightarrow \infty$

$$
\alpha_{\mathbf{p}}(\mathbf{r} \rightarrow \infty)=\theta(z),
$$

which is derived from (6). 
We write the particular solution $\delta g_{\mathbf{p}}^{K}(\mathbf{r}, \varepsilon)$ of a nonhomogeneous equation (5) using the corresponding Green's function $g_{\mathbf{p} \mathbf{p}^{\prime}}\left(\mathbf{r}, \mathbf{r}^{\prime}\right)=g_{-\mathbf{p}^{\prime},-\mathbf{p}}\left(\mathbf{r}^{\prime}, \mathbf{r}\right)$ :

$$
\delta g_{\mathbf{p}}^{K}(\mathbf{r})=\int d \mathbf{p}^{\prime} d \mathbf{r}^{\prime} g_{\mathbf{p p}^{\prime}}\left(\mathbf{r}, \mathbf{r}^{\prime}\right) I_{i}^{*}\left(g_{\mathbf{p}}^{(0) K}\right)
$$

The function $g_{\mathbf{p p}^{\prime}}$ should be determined from the relations

$\mathbf{v} \frac{\partial}{\partial \mathbf{r}} g_{\mathbf{p} \mathbf{p}^{\prime}}\left(\mathbf{r}, \mathbf{r}^{\prime}\right)-I_{i}\left(g_{\mathbf{p \mathbf { p } ^ { \prime }}}\right)=\delta\left(\mathbf{p}-\mathbf{p}^{\prime}\right) \delta\left(\mathbf{r}-\mathbf{r}^{\prime}\right)$,

$$
g_{\mathbf{p \mathbf { p } ^ { \prime }}}\left(\mathbf{r}, \mathbf{r}^{\prime} \rightarrow \infty\right)=0
$$

Substituting the value of $\delta g_{\mathbf{p}}^{K}$ in Eq. (1), we obtain the expression for the change in the electric current $\Delta I$ due to specific defects

$$
\begin{gathered}
\Delta I=-\frac{\pi e}{2} N(0) \int d S \int \frac{d \varepsilon}{2 \pi}\left\langle v_{z} \delta g_{\mathbf{p}}^{K}(\mathbf{p})\right\rangle= \\
=-\frac{\pi e}{2} N(0) \int \frac{d \varepsilon}{2 \pi} \int d \mathbf{p} \int d \mathbf{r} G_{\mathbf{p}}(\mathbf{r}) I_{i}^{*}\left(g_{\mathbf{p}}^{(0) K}(\mathbf{r})\right),
\end{gathered}
$$

where

$$
G_{\mathbf{p}}(\mathbf{r})=\int_{S} d^{2} \boldsymbol{\rho} \int d \mathbf{p}^{\prime} v_{z}^{\prime} g_{\mathbf{p}^{\prime} \mathbf{p}}(\boldsymbol{\rho}, \mathbf{r})
$$

Multiplying Eq.(11) by $v_{z}$ and integrating it by $\rho$ over the contact area and momentum $\mathbf{p}$, we obtain the following equation for the function $G_{\mathbf{p}}(\mathbf{r})$ :

$$
\mathbf{v} \frac{\partial}{\partial \mathbf{r}} G_{\mathbf{p}}(\mathbf{r})+I_{i}\left(G_{\mathbf{p}}(\mathbf{r})\right)=-\delta(z) .
$$

The probability $\alpha_{\mathbf{p}}(\mathbf{r})$ satisfies Eq. (8), which combined with Eq.(15) yields the relation [15]

$$
G_{\mathbf{p}}(\mathbf{r})=\alpha_{-\mathbf{p}}(\mathbf{r})-\theta(z) .
$$

Substituting (7) and (16) into the expression for the point-contact current correction (13), we find

$$
\begin{aligned}
\Delta I=-\pi e N(0) & \int \frac{d \varepsilon}{2 \pi}\left\{G_{0}\left(\varepsilon+\frac{e U}{2}\right)-G_{0}\left(\varepsilon-\frac{e U}{2}\right)\right\} \times \\
& \times \int d \mathbf{r} \int d \mathbf{p} \alpha_{-\mathbf{p}}(\mathbf{r}) I_{i}^{*}\left(\alpha_{\mathbf{p}}(\mathbf{r})\right) .
\end{aligned}
$$

Relation (17) has a wide range of applications. It is valid for any mean free path as to the electron scattering on background impurities $l_{i}$ in case their separation distance $r_{0}$ is much smaller than the contact diameter $d$, and also for arbitrary relation between the value $d$ and the distance between the specific defects $r_{0}^{*}$, which provide the mean free path for electron scattering on these defects, $l_{i}^{*}>d, l_{i}$.

The following calculations depend on the properties of both types of scatterers and their concentrations. The integrodifferential equation for the probability $\alpha_{p}$ (8) can be solved analytically for extreme cases in which the mean free path $l_{i}$ is much greater or much smaller than the diameter $d$. For the ballistic regime of electron motion through the contact we obtain

$$
\alpha_{\mathbf{p}}(\mathbf{r})= \begin{cases}\theta(-z)+\operatorname{sign} z, & -\mathbf{v} \in \Omega(\mathbf{r}) \\ \theta(-z), & -\mathbf{v} \notin \Omega(\mathbf{r}), \quad l_{i}>>d\end{cases}
$$

$\Omega(\mathbf{r})$ is a solid angle at which the aperture is seen from the point $\mathbf{r} ; \theta(z)$ is the (Heaviside) unit step function.

For the diffusive regime of the electron motion through the contact $\alpha_{\mathbf{p}}$ can be expanded in a series in a small relaxation time $\tau_{i}$ (Ref. 15)

$$
\alpha_{\mathbf{p}}(\mathbf{r})=\left\langle\alpha_{\mathbf{p}}(\mathbf{r})\right\rangle-\tau_{i} \mathbf{v} \nabla\left\langle\alpha_{\mathbf{p}}(\mathbf{r})\right\rangle+\ldots \quad l_{i}<d,
$$

where

$$
\begin{gathered}
\left\langle\alpha_{\mathbf{p}}(\mathbf{r})\right\rangle=\theta(z)-\operatorname{sign} z \frac{1}{\pi} \arctan \frac{1}{Q} \\
Q^{2}=\frac{2 r^{2}}{d^{2}}-\frac{1}{2}+\left[\left(\frac{2 r^{2}}{d^{2}}-\frac{1}{2}\right)^{2}+\frac{4 z^{2}}{d^{2}}\right]^{1 / 2} .
\end{gathered}
$$

The solution of Eq. (4) for the scattering matrix is complicated and can be found only in some special cases. For example, if the interaction with a single impurity located at $\mathbf{r}_{i}$ is described by the spin-independent operator $\hat{V}(\mathbf{r})=V \hat{I} \delta\left(\mathbf{r}-\mathbf{r}_{i}\right)(\hat{I}$ is a singular matrix, $V=$ const), then for an arbitrary interaction potential $V$ the $\hat{t}$ matrix will have the form

$$
\hat{t}=\frac{\hat{V} \hat{I}-i \pi V^{2}\left\langle\hat{g}_{\mathbf{p}}^{(0)}\right\rangle N(0)}{1+[\pi N(0) V]^{2}} \delta\left(\mathbf{r}-\mathbf{r}_{i}\right) .
$$

In the case of a scattering center with nonzero spin $\mathbf{s}$ the electron interaction energy with such a scatterer contains the component $\hat{V}=J \hat{\sigma} \hat{\mathbf{s}} \delta\left(\mathbf{r}-\mathbf{r}_{i}\right)$ ( $\hat{\boldsymbol{\sigma}}$ is an electron spin operator, and $J$ is an exchange interaction constant). At temperatures $T$ above the Kondo temperature $T_{K} \sim \varepsilon_{F} \exp (-1 / N(0) J)$ we can construct the Born series for the scattering matrix. Standard calculations [16] in the second Born approximation give 


$$
t^{K}=\frac{i}{2} \Gamma(\mathbf{r}, \varepsilon)\left\langle g_{\mathbf{p}}^{(0) K}\right\rangle ; t^{R, A}=\int \frac{d \varepsilon}{2 \pi} \frac{\Gamma(\mathbf{r}, \varepsilon)}{\varepsilon-\varepsilon^{\prime} \pm i 0},
$$

where $\quad \Gamma(\mathbf{r}, \varepsilon)=\pi N(0) J^{2} s(s+1) \times$

$$
\times\left[1-\frac{J N(0)}{2} \int d \varepsilon^{\prime} \frac{\left\langle g_{\mathbf{p}}^{(0) K}\right\rangle}{\varepsilon-\varepsilon^{\prime}}\right] \delta\left(\mathbf{r}-\mathbf{r}_{i}\right) .
$$

If we have $N>>1$ specific defects which yield an electron mean free path $l_{i}^{*}>>\max \left(d, l_{i}\right)$, the calculation of the current through the contact can be performed by ignoring the correlation between the individual scattering events and writing the operator $\hat{t}$ of the system in the form of the sum of $\hat{t}$-operators of scattering by isolated defects. In this approximation, in the case of isotropic scattering by zero-spin impurities the resistance $R$ of a point contact has the form

$$
\begin{aligned}
& R^{-1}=R_{c}^{-1}\left\{1-\frac{\sigma^{*}}{S} \sum_{\mathbf{r}_{i}} M\left(\mathbf{r}_{i}\right)\right\} \\
& R_{c}^{-1}=e^{2} N(0) \int_{S} d^{2} \rho\left\langle v_{z} \alpha_{\mathbf{p}}(\rho)\right\rangle ;
\end{aligned}
$$

$R_{c}$ is the contact resistance without the specific impurities [13],

$$
\sigma^{*}=\frac{\operatorname{Im} t^{A}}{v_{F}}=\frac{\pi V^{2} N(0)}{v_{F}\left[1+(\pi N(0) V)^{2}\right]}
$$

is the effective cross section of scattering by the specific impurities.

$$
M\left(\mathbf{r}_{i}\right)=4 \frac{R_{c}}{R_{0}}\left\langle\alpha_{-\mathbf{p}}\left(\mathbf{r}_{i}\right)\left[\left\langle\alpha_{\mathbf{p}^{\prime}}\left(\mathbf{r}_{i}\right)\right\rangle-\alpha_{\mathbf{p}}\left(\mathbf{r}_{i}\right)\right]\right\rangle ;
$$

$R_{0}^{-1}=1 / 2 e^{2} v_{F} N(0) S$, and $R_{0}$ is the resistance of a ballistic contact (Sharvin formula) $[10,11]$.

The sum of the $M\left(\mathbf{r}_{i}\right)$ functions depends on the specific configuration of the impurities and describes the classical mesoscopic effects in the conductivity of point contacts. For the ballistic case in which the function $\alpha_{\mathbf{p}}$ is determined by Eq.(18), we have [9]

$$
M\left(\mathbf{r}_{i}\right)=\left[\frac{\Omega\left(\mathbf{r}_{i}\right)}{2 \pi}\right]^{2} .
$$

Figure 2 shows the results of a numerical calculation for Eqs. (24) and (27). In the mesoscopic region $\left(d \sim r_{0}^{*}\right)$ the behavior of the resistance is essentially determined by the presence of impurities at distances $r_{i} \sim d$ from the aperture. If there are some impurities, then we will have $N$-shaped de-

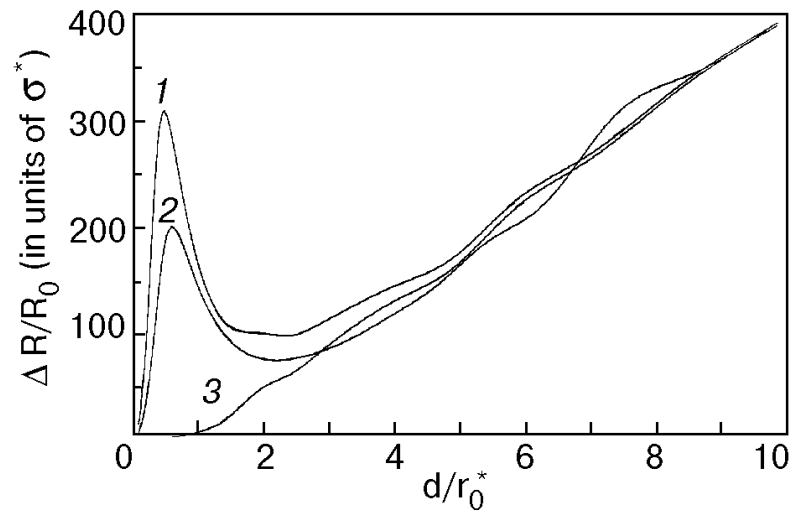

Fig. 2. The dependence of the relative correction $\Delta R / R_{0}$ for the ballistic resistance of the point contact $R_{0}$ versus the ratio between its diameter and the characteristic distance $r_{0}^{*}$ between the defects for three random distributions in the contact region. Curves 1 and 2 represent the cases in which one of the impurities is located in the contact region, and curve 3 is the case in which there is no such impurity present.

pendences $\left(\Delta R / R_{0}\right)(d)$ (curves 1 and 2 in Fig. 2); otherwise, we have a monotonic $\left(\Delta R / R_{0}\right)(d)$ dependence (curve 3 in Fig. 2). It should be noted that while $d / r_{0}^{*}$ is increased from some point, the value of $\Delta R / R_{0}$ stays below its asymptotic value:

$$
\frac{\Delta R}{R_{0}}=\frac{16}{3 \pi} n_{i}^{*} \sigma^{*} d
$$

Figure 3 illustrates a situation that might be observed in experiments [12] when the defects are concentrated in a thin layer near the contact. In such a case the contaminated region plays the role of a barrier with effective transparency $D$. It is known [17] that the relative change $\Delta R / R_{0}$ in the resistance of a ballistic contact due to the translu-

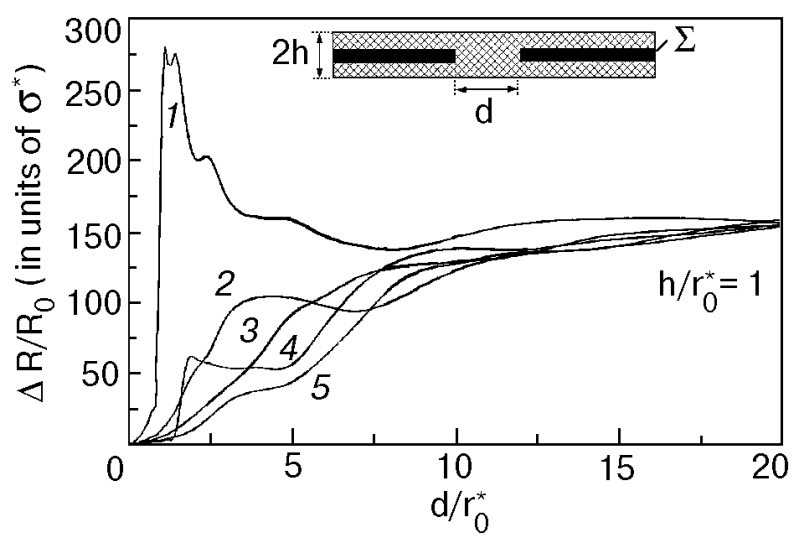

Fig. 3. Relative correction $\Delta R / R_{0}$ for the resistance of a ballistic contact for the various spatial distributions of impurities when the defects are located in a thin layer in the insulating plane (curves 2-5). Curve 1 represents a rare case in which one of the impurities initially appears in the near contact region. 
cent barrier is $1-D$ and does not depend on the diameter $d$. The assumption made is supported by the results of a numerical calculation (Fig. 3), which shows a fairly weak $\left(\Delta R / R_{0}\right)(d)$ dependence for contact sizes, of the order of the thickness of a layer in which the scatterers are concentrated.

For contacts with a small mean free path, using Eqs. (19) and (26), we obtain

$$
R_{c}^{-1}=R_{M}^{-1}=\frac{2}{3} e^{2} v_{F} N(0) l_{i} d ;
$$

$R_{M}$ is the resistance of the diffusion contact (Maxwell's equation);

$$
M\left(\mathbf{r}_{i}\right)=\frac{l_{i}}{\pi d} \frac{1}{\left(Q^{2}+1\right)\left(Q^{2}+4 z_{i}^{2} /\left(Q^{2} d^{2}\right)\right)},
$$

where $Q^{2}\left(\mathbf{r}_{i}\right)$ is defined by Eq. (21). If the characteristic distance between the specific scatterers $r_{0}^{*}$ is much larger than the contact size $r_{0}^{*}>d$, the impurities located at distances $r_{i} \gg d$ from the orifice yield $M\left(\mathbf{r}_{i}\right) \simeq\left(l_{i} / d\right)\left(S^{2} / r_{i}^{4}\right)$. Separating the contribution of distant defects, we obtain for a relative correction to the resistance

$$
\frac{\Delta R}{R_{M}} \simeq \frac{\sigma^{*}}{S} \sum_{r_{i} \leqslant d} M\left(\mathbf{r}_{i}\right)+\frac{l_{i}}{l_{i}^{*}} \frac{S}{r^{*} d},
$$

where $l_{i}^{*-1}=\sigma^{*} n^{*}, l_{i}=v_{F} \tau_{i}$, and $r^{*} \approx r_{0}^{*}$. The first term in Eq. (31) is the contribution of specific defects in the contact region to the contact resistance. The partial contribution of every scatterer to the $\Delta R$ value is of the order of $\sigma^{*} d / S l_{i}$ and increases as the diameter is reduced. In a real situation for $r_{0}^{*}>>d$ there might be no defects at distances $r_{i} \leqslant d$ from the contact, and the relation $\Delta R / R_{0}$ is proportional to $d$.

Now if $d>r_{0}^{*}$, the location of defects has no effect on the resistance of a point contact. Such inequality allows us to switch in (24) from a sum over the impurity coordinates $r_{i}$ to an integral over the whole space. This yields for the circular contact

$$
R^{-1}=R_{M}^{-1}\left(1-l_{i} / l_{i}^{*}\right)
$$

Note that Eq. (32) can be directly obtained from expression (29) for the resistance of a short mean free path contact in which, according to Matthiessen's rule, $l_{i}^{-1}$ should be replaced by $l_{i}^{-1}+l_{i}^{*-1}$, bearing in mind that $l_{i}<<l_{i}^{*}$.

The $\left(\Delta R / R_{M}\right)(d)$ dependence, which is determined by analytical equations (31) and (32), is illustrated in Fig. 4, showing the results of the numerical calculation using Eqs. (24) and (30) for various random distributions of the specific scatterers. The

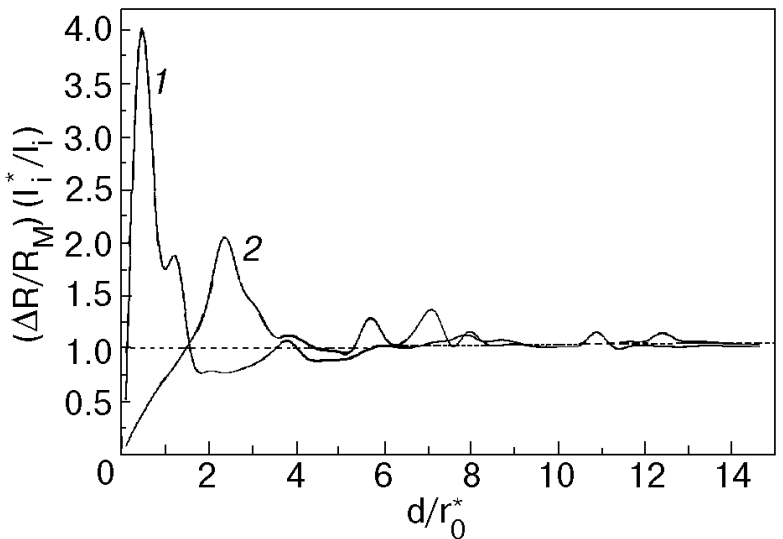

Fig. 4. Dependence of the relative correction $\Delta R / R_{M}$ for the resistance of a contact with small mean free path on the ratio between its diameter $d$ and the characteristic distance between the specific defects $r_{0}^{*}$. Curves 1 and 2 correspond to two different distributions of these defects.

mesoscopic regime in the conductance of point contacts is observed up to the values of $d / r_{0}^{*}$ of about $5-10$

For the electron scattering by specific impurities with nonzero spin, substituting expression (23) for $t^{A}$ into (17) at temperatures above the Kondo temperature, we can formally write the differential resistance of a point contact in the form (24), denoting by $\sigma^{*}$ the following function of the voltage and temperature:

$$
\sigma^{*}=\frac{\pi N(0) J^{2} s(s+1)}{2 v_{F}}\left[1-\frac{J N(0)}{4} \frac{d F(e U)}{d e U}\right],
$$

where

$$
\begin{aligned}
& F(e U)=\int_{0}^{\infty} d \varepsilon \varepsilon G_{0}(\varepsilon) \int_{0}^{\infty} \frac{d \varepsilon^{\prime}}{\varepsilon^{2}-\varepsilon^{\prime 2}} \times \\
& \times\left[G_{0}\left(\varepsilon^{\prime}-e U\right)-G_{0}\left(\varepsilon^{\prime}+e U\right)\right] .
\end{aligned}
$$

The second term in square brackets of expression (33), which is a function of voltage and temperature, describes the Kondo anomaly on the currentvoltage characteristic of the point contact. Note that the value $\sigma^{*}$, evaluated in the second Born approximation, does not depend on the scattering by background defects, and Eq. (33) is valid for any relation between the contact diameter $d$ and the mean free path $l_{i}$.

Therefore, the increase in concentration of the specific impurities $n_{i}^{*}$, which leads to the alteration of the ratio between the characteristic separation of the impurities $r_{0}^{*}=\left(n_{i}^{*}\right)^{-1 / 3}$ and the contact diame- 
ter, changes the size-dependence of the resistance of a point contact, $R(d)=R_{M}+\Delta R$ :

$$
\frac{\Delta R}{R_{M}} \sim \begin{cases}l_{i} / l_{i}^{*}, & r_{0}^{*}<<d \quad l_{i}^{*}<<d<<l_{i}, \\ \frac{d l_{i}}{r_{0}^{*} l_{i}^{*}}+\frac{N_{0} \sigma^{*}}{d l_{i}^{*}}, & r_{0}^{*}>>d,\end{cases}
$$

where $N_{0}$ is the number of defects in contact region. The influence of specific defects on the resistance for low concentrations $\left(r_{0}^{*}>>d\right)$ is determined by their spatial distribution, which leads to diverse (random) dependences $\Delta R(d)$. They can be split into two groups: 1) $\left(\Delta R / R_{M}\right)(d)$ increases with decreasing $d$ if at least one of the specific impurities is located at a distance $r<d$ from the contact aperture; 2$)$ the ratio $\left(\Delta R / R_{M}\right)$ decreases with decreasing $d$ if all impurities are located far enough from the contact. As mentioned above, the total contribution from all defects at different distances from the contact can lead to the appearance of a minimum on the curve $\left(\Delta R / R_{M}\right)(d)$. For high concentrations $\left(r_{0}^{*}<<d\right)$ the relative change in resistance is independent of the contact size, $\Delta R / R_{M}=\operatorname{const}(d)$. Thus the functional dependence of the point contact resistance versus its diameter can serve as a criterion for determining the concentration of the specific impurities, and also their distribution in the contact region. Note that due to the «screening» effect of background impurities the classical mesoscopic effect in the diffusive contact is weakened $d / l_{i}>1$ times compared to the ballistic case described in Ref. 7. If the specific impurities are magnetic impurities, they lead to a nonlinear dependence of the correction for the contact resistance versus the ap- plied voltage. Such dependence can be extracted in standard experiments in point-contact spectroscopy. In this case the distribution of impurities in the contact region affects the intensity and displacement (on the voltage scale) of the Kondo anomaly in the point contact spectrum.

1. C. J. Muller, J. M. van Ruitenbeek, and L. J. de Jongh, Physica C191, 485 (1992).

2. E. N. Bogachek, A. N. Zagoskin, and I. O. Kulik, Sov. J. Low Temp. Phys. 18, 796 (1990).

3. J. M. Krans, J. M. van Ruitenbeek, V. V. Fisun, I. K. Yanson, and L. J. de Jongh, Nature 375, 767 (1995).

4. P. A. M. Holweg, J. A. Kokkedee, J. Caro, A. H. Vebruggen, S. Radelaar, A. G. M. Jansen, and P. Wyder, Phys. Rev, Lett. 67, 2549 (1991).

5. V. I. Kozub, J. Caro, and P. A. M. Holweg, Phys, Rev. B50, 15126 (1994)

6. E. G. Haanappel and D. van der Marel, Phys. Rev. B39, 5484 (1989).

7. A. M. Zagoskin, S. N. Rashkeev, R. I. Shekhter, and G. Wendin, J. Phys.: Cond. Matt. 7, 6253 (1995).

8. Yu. M.Gal'perin and V. I. Kozub, Sov. J. Low Temp. Phys. 18, 336 (1992)

9. Yu. A. Kolesnichenko, A. N. Omelyanchouk, and I. G. Tuluzov, Low Temp. Phys. 21, 655 (1995).

10. Yu. V. Sharvin, Sov. Phys. JETP 21, 655 (1965).

11. I. O. Kulik, A. N. Omelyanchouk, and R. I. Shekhter, Sov. J. Low Temp. Phys. 3, 740 (1977).

12. I. K. Yanson, V. V. Fisun, R. Hesper, A. V. Khotkevich, J. M. Krans, J. A. Mydosh, and J. M. Ruitenbeek, Phys. Rev. Lett. 74, 302 (1995).

13. L. V. Keldysh, Sov. Phys. JETP 20, 1018 (1964).

14. G. Eilenberger, Z. Phys. 214, 195 (1968).

15. I. O. Kulik, R. I. Shekhter, and A. G. Shkorbatov, Sov. Phys. JETP 54, 1130 (1981).

16. Yu. M. Ivanchenko and G. A. Trush, Sov. Phys. JETP 67, 352 (1974)

17. R. I. Shekhter and I. O. Kulik, Sov. J. Low Temp. Phys. 9, 46 (1983). 